\title{
Registro del Piquero Pardo Sula leucogaster (Aves: Suliformes: Sulidae) en el Refugio de Vida Silvestre Isla Santa Clara, Ecuador
}

\author{
Teddy Ochoa P ${ }^{1}$, César Garzón Santomaro ${ }^{2}$ \\ ${ }^{1}$ Ministerio de Ambiente, Dirección del Ambiente de la provincia de El Oro, Av. Vela entre 25 de Junio y \\ Av. Sucre, Machala, Ecuador. \\ ${ }^{2}$ Instituto Nacional de Biodiversidad, División de Ornitología, pasaje Rumipamba 341 y Av. de los Shyris, \\ Casilla Postal 17-07-8976. Quito, Ecuador. \\ * Corresponding autor. E-mail: cesar.garzon@biodiversidad.gob.ec
}

\section{Resumen}

Reportamos un nuevo registro de distribución de Piquero Pardo Sula leucogaster (Sulidae) en el Refugio de Vida Silvestre de la Isla de Santa Clara, en el Golfo de Guayaquil, $43 \mathrm{~km}$ de Puerto Bolívar, Machala, provincia de El Oro. Fue observado en un período de 10 días entre el 7 al 17 de julio de 2016, cerca del centro de interpretación ambiental de la isla. Se observó una hembra cerca de un grupo de Piqueros Patas Azules (Sula nebouxii), que la desplazaron a otra zona de isla. No existen reportes previos publicados de esta especie en la parte suroccidental del Ecuador.

Palabras clave: Ave marina, especie pantropical, isla, distribución

\section{Abstract}

We report a new distribution record of the Brown Booby Sula leucogaster (Sulidae) in the Island of Santa Clara Wildlife Refuge, Gulf of Guayaquil, $43 \mathrm{~km}$ from Puerto Bolivar, Machala, province of El Oro. It was observed over a 10-days period between 7 to July 17,2016 , near the environmental interpretation center. A female was first observed near a group of Blue-Footed Boobies (Sula nebouxii), but it was later displaced to a different part of the island. There are no previous published report for this species in Ecuador.

Keywords: Seabird, pantropical species, island, distribution

Editado por /

Edited by:

Diego F. Cisneros -

Heredia

Recibido /

Received:

2016/10/06

Aceptado /

Accepted:

2018/06/21

Publicado en línea / Published online: 2018/12/25 
El Piquero Pardo Sula leucogaster (Boddaert, 1783) es un ave marina común en aguas tropicales de todo el mundo. Su distribución se describe como pantropical y se extiende en el océano Pacífico desde las costas norte desde la península de California llegando hasta el sur en las costas de Panamá y Colombia [1-2]. También se distribuye en el océano Atlántico, Índico, en el mar Caribe, mar Rojo y en los mares al norte de Australia [1]. Esta especie presenta una gran capacidad de dispersión y se le ha registrado más allá de su rango de distribución, con individuos reportados en diferentes estados de la costa oeste de los Estados Unidos [3-5], en Columbia Británica [6] y en Alaska [7]. Los registros más al sur han sido reportados a 19,3km de las costas de Perú en la Isla Huampanú [8] y en los islotes al suroccidente de la Isla de Pascua [9].

Esta especie pelágica es considerada como un visitante muy raro en aguas abiertas a lo largo de la costa norte de Ecuador [10]. Los registros en Ecuador de esta especie incluyen individuos observados en junio 2000 en la Isla de la Plata, provincia de Manabí [11]; en diciembre 2008, a 57,4km al norte de Punta Galeras, provincia de Esmeraldas [12]; y en junio 2010, cerca de la Chocolatera, en la provincia de Santa Elena [13]. Según McMullan y Navarrete [14] es una especie muy rara en las costas del noroccidente de la provincia de Esmeraldas. En la presente contribución presentamos un nuevo registro de esta especie en Ecuador.

El 7 julio del 2016 a las 18:15, observamos un Piquero Pardo en la Isla Santa Clara, a $47 \mathrm{~km}$ de la costa ecuatoriana, en la provincia de El Oro (Fig. 1), en la parte baja cerca del campamento de guardaparques ( -3.173460,-80.636058). Este reporte constituye el primer registro documentado de la presencia de esta especie en la Isla de Santa Clara y además la observación más al sur de Ecuador a 125km de la Chocolatera en la provincia de Santa Elena. El piquero observado (Fig. 2) presentaba las características de una hembra adulta debido a su plumaje marrón oscuro en la cabeza, cuello, alas y espalda, mientras que su vientre es de color blanco, pico y patas verde amarillento, ala ventral blanco [2]. El macho difiere de la hembra por presentar la cabeza y cuello de gris a marrón, más pálido que la espalda, alas y cola [2]. Según estas características y el rango distribución de esta especie se puede tratar de la subespecie S. I. etesiaca [15], cuya distribución es la zona muy húmeda del Pacífico oriental, donde el límite más extremo sur es el cabo San Francisco al norte de Ecuador y el norte no está bien conocido, pero se extiende seguramente hasta las costas de México [2]. Sula leucogaster leucogaster difiere de S. I. etiesiaca por tener la cabeza y el cuello claramente más oscuros que la espalda, las alas y la cola [16]. La subespecie S. I. brewslei tiende a tener la cabeza de color gris claro a blanco que poco a poco se vuelve marrón en el cuello y la parte superior de la espalda más oscura que otras especies de S. leucogaster [17]. Sula leucogaster plotus tienen las partes superiores, el cuello y la garganta de color castaño oscuro claramente cortadas en la parte superior del pecho, el resto de las partes inferiores de color blanco, y el castaño oscuro se junta al marrón del borde delantero del ala [16]. 

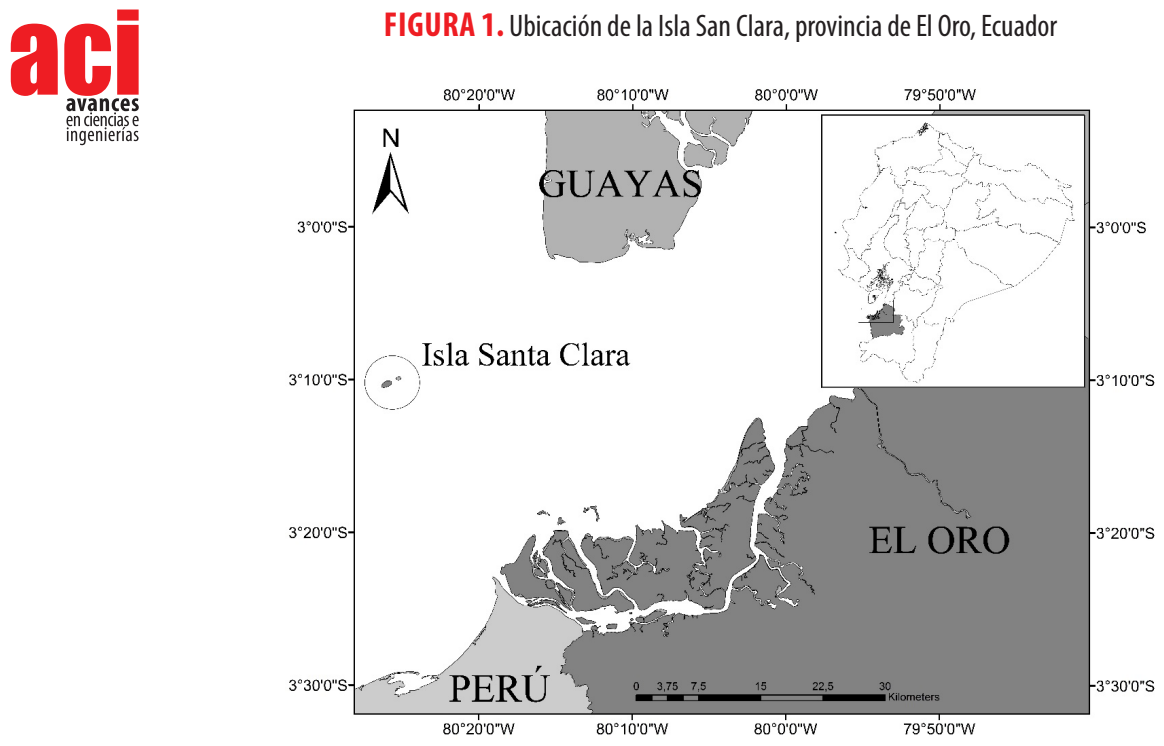

La primera vez que observamos el Piquero Pardo estaba perchado en medio de una colonia de Piqueros Patas Azules Sula nebouxii (Fig. 3). Permaneció durante una hora en ese lugar, después el grupo de piqueros se comportó de forma hostil contra el Piquero Pardo, siguiéndole y picoteándole hasta desplazarle a la parte alta de la estructura donde se está construyendo el centro de interpretación (Fig. 4). Este comportamiento agresivo es normal entre individuos de la misma especie de Piquero Patiazul y se relacionado con su territorialidad en el limitado espacio de la isla.

Luego de la primera observación, esta especie fue monitoreada todos los días, desde el 7 de julio hasta su último registró el 17 de julio. El Piquero Pardo permaneció en la Isla Santa Clara durante 10 días, siempre en el mismo sitio aproximadamente las 06h00 hasta $07 \mathrm{~h} 45$ y desde las 17h45 hasta el anochecer. Las horas en que no era registrado en la isla posiblemente el Piquero Pardo se encontraba alimentándose en el mar o cerca de los barcos pesqueros, regresando a la isla al atardecer para descansar. Después de la última observación se efectuaron recorridos alrededor de la isla para confirmar su ausencia o presencia. Durante el tiempo de permanencia del Piquero Pardo en la isla no se observó comportamiento agresivo con otras especies.

Durante los registros realizados Isla Huampanú (Perú) y en las islas de La Plata y Santa Clara (Ecuador), S. leucogaster se observó cerca de individuos de Piquero Peruano S. variegata, de Piquero de Nazca S. grantii y S. nebouxii. Por esta razón, en futuras observaciones se recomienda poner mucha atención a las colonias de piqueros, debido a que puede registrar nuevamente al Piquero Pardo, especialmente en las islas alejadas de la costa ecuatoriana. 


\section{AGRADECIMIENTOS}

Los autores agradecemos a los dos técnicos del área protegida: Blgo. José Luis Mena y al Per. For. Edwin Sánchez por revisar y comentar esta nota científica.

FIGURA 2. Características del Piquero Pardo S. leucogaster Teddy Ochoa P., 2016



FIGURA 3. Observación del Piquero Pardo S. leucogaster en medio de Piquero Patiazul S. nebouxii. Teddy 0choa P., 2016

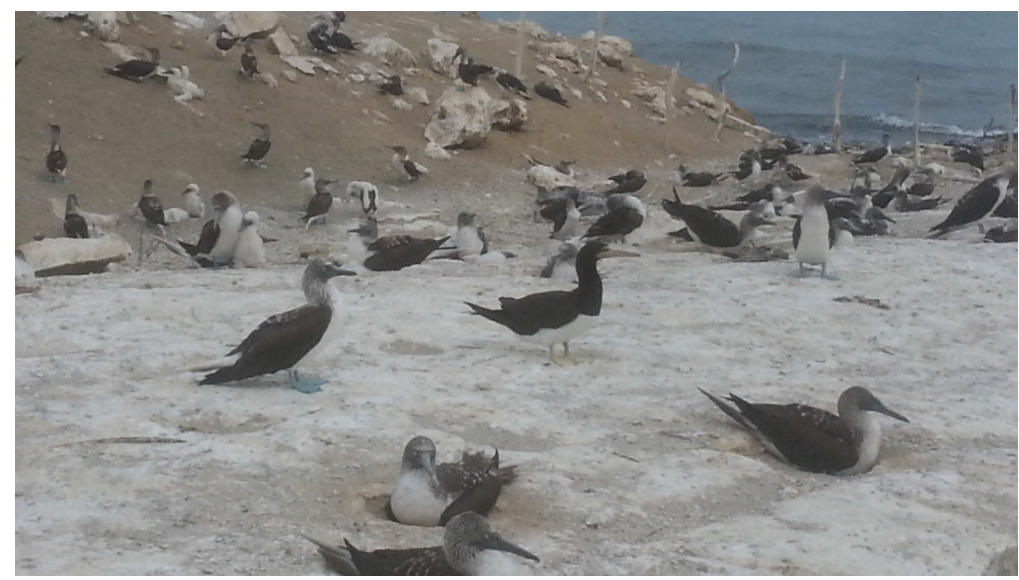


FIGURA 4. Sula leucogaster desplazado a la parte alta de la estructura en donde se está construyendo el centro de interpretación ambiental de la Isla Santa Clara. Teddy Ochoa P., 2016

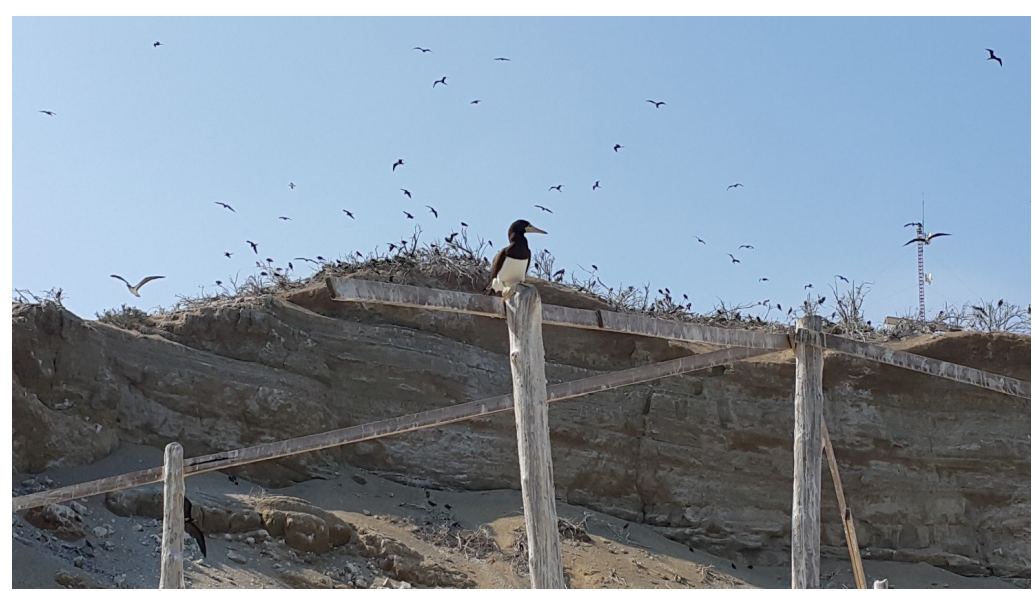




\section{REFERENCIAS}

[1] Schreiber, E. A., y Norton, R. L. (2002). Brown Booby: Sula leucogaster. Birds of North America, Incorporated.

[2] Murphy, R. C. (1936). Oceanic birds of. South America.'The American Museum of Natural History, New York.

[3]. Ceyca, J. P., y Mellink, E. (2009). Ecología reproductiva del bobo café (Sula leucogaster) en Morros el Potosí, Guerrero, México. Revista mexicana de biodiversidad, 80(3), 793-800.

[4] Oregonbirds.org. (2012). Oregon Field Ornithologists - OFO. Recuperado el 30 de septiembre de 2016 de: http://www. oregonbirds.org/index.html.

[5] Wahl, T.; Tweit, B.; Mlodinow, S. (2005). Birds of Washington: Status and Distribution. Oregon State University Press, Corvallis, Oregon. 436pp.

[6] Morgan, K., Wallace, S., y Krause, G. (2009). First record of a Brown Booby in British Columbia, Canada. British Columbia Birds, 19, 13-15.

[7] Gibson, D.; DeCicco, L.; Gill Jr. R.; Heinl, S.; Lang, A.; Tobish Jr, G.; Withrow, J. (2015). Checklist of Alaska Birds. 21st Edition: 2015. Recuperado el 4 de agosto de 2016 de: http://www.universityofalaskamuseumbirds.org/ products/checklist.pdf.

[8] Valverde, M. (2007). Registro del Piquero Pardo Sula leucogaster en una isla del Perú. Biologist (Lima). Volumen $5(2): 65,67$.

[9] Flores, M., Lazo, P., \& Hucke-Gaete, R. (2013). Estado del conocimiento de los piqueros pantropicales (Sulidae) en las islas oceánicas chilenas y primer registro de piquero café Sula leucogaster en Isla de Pascua. Revista de biología marina y oceanografía, 48(3), 667-672.

[10] Ridgely, R. S., Greenfield, P. J., Coopmans, P., \& Kalil, G. (2006). Aves del Ecuador: Guía de campo. Fundación de Conservación Jocotoco. Volumen 2. Academia de Ciencias Naturales de Filadelfia y Fundación de Conservación Jocotoco.

[11] Cisneros-Heredia, D. F. (2005). La avifauna de la isla de La Plata, Parque Nacional Machalilla, Ecuador, con notas sobre nuevos registros. Cotinga, 24, 22-27.

[12] Agreda A.; Torres, S.; Hasse, B.; y Samaniego, J. (2011). Investigaciones de la avifauna marina en aguas continentales ecuatorianas con énfasis en su distribución, diversidad, abundancia y estado de conservación. Acta Oceanográfica del Pacífico Volumen 16 (1): 2010-2011

[13] Haase, B. J. M. (2011). Aves marinas de Ecuador continental y acuáticas de las piscinas artificiales de Ecuasal. Aves \& Conservación, BirdLife International and Ecuasal SA: Guayaquil.

[14] McMullan, M., y Navarrete, L. (2013). Fieldbook of the Birds of Ecuador: including the Galápagos Islands. Fundación de conservación Jocotoco.

[15] Nelson, B. (1978). The Sulidae: gannets and boobies (No. 154). Oxford University Press.

[16] Redman, N.; Stevenson, T.; Fanshawe, J. (2011). Birds of the Horn of Africa. Ethiopia, Eritrea, Djibouti, Somalia and Socotra. Ilustrations by John Gale and Brian Small. Second edition published 2011. Imprint of Boomsburry Publishing PIC, 50 Bedford Square, London, WC1B 3DP.

[17] Langteau, J. (2017). Sula leucogaster (brown booby). Animal Diversity Web. Recuperado el 22 de enero de 2017 de: https://animaldiversity.org/accounts/Sula_leucogaster/ 